\title{
Are molecular target therapies limited by cardiotoxicity - causes and symptoms of cardiovascular damage
}

\author{
Beata Jagielska ${ }^{1}$, Konrad Tałasiewicz ${ }^{1}$, Aleksandra Czachowska ${ }^{1}$, Katarzyna Gepner ${ }^{1}$, \\ Elżbieta Mądro², Małgorzata Symonides ${ }^{3}$, Paweł Wiechno ${ }^{4}$, Maciej Krzakowski ${ }^{5}$
}

\begin{abstract}
Since the introduction of new drugs, (commonly referred to as 'Molecular Target Therapies'), into oncological clinical practice both the number of objective indicators/endpoints of achieved treatment response and cancer survival duration have increased. Nevertheless, the risk of cardiovascular complications has also risen. Optimistic reports on the relatively low cardiotoxicity of these drugs have been verified through experience. Routine clinical practice has witnessed growing numbers of new drug groups that have different molecular target points and also a varied cardiotoxicity.

This paper presents the most important cardiovascular complications associated with the use of molecularly targeted drugs and includes their mechanisms of development.
\end{abstract}

NOWOTWORY J Oncol 2017; 67, 1:34-40

Key words: cardiotoxicity, molecular target therapy, arterial hypertension, cardiac failure, arrhythmias, drug side-effects

\section{Introduction}

Administering molecularly targeted systemic cancer treatment represents a new and valued benefit in oncology. An important element to this approach is recognising that certain genetic abnormalities are associated with malignant transformation, growth factors and their receptors. The proliferation and differentiation of malignant cells caused by the triggering of the 'molecular pathway' arises from activation of membrane receptors. When modified, these molecular mechanisms may allow prolonged progression-free survival and also in some cancer patients, their overall survival. Nevertheless, despite the optimistic toxicity profiles reported initially, it became apparent very soon that many were associated with adverse reactions (including significant cardiac toxicity) making their withdrawal necessary or even resignation from the anti-cancer treatment [1]. It must of course be remembered that such drugs are often given to patients, who had been previously treated with conventional chemotherapy and which had also affected their cardiovascular system [2]. Furthermore, a significant number of cancer patients suffered from concomitant diseases. Data from the National Cancer Registry shows that cancer develops over the age of 60 years in $70 \%$ of men and in $60 \%$ of women, and is most common in the eighth decade of life; this being very well illustrated in prostate cancer cases [3]. In some clinical settings, just a solitary

\footnotetext{
${ }^{1}$ Department of Oncology Diagnostics, Cardioncology and Palliative Medicine, The Maria Skłodowska-Curie Memorial Cancer Center and Institute, Warszawa, Poland

${ }^{2}$ Institute of Hematology and Transfusion Medicine, Warszawa, Poland

${ }^{3}$ Department of Anesthesiology and Intensive Care, The Maria Skłodowska-Curie Memorial Cancer Center and Institute, Warszawa, Poland

${ }^{4}$ Department of Urinary Tract Malignacies, The Maria Skłodowska-Curie Memorial Cancer Center and Institute, Warszawa, Poland

${ }^{5}$ Department of Lung \& Thoracic Tumours, The Maria Skłodowska-Curie Memorial Cancer Center and Institute, Warszawa, Poland
} 
characteristic of the tumour (for instance its localisation) may cause cardiovascular disturbances.

Within this context tumours of the heart are of minor importance; both primary and secondary. The former are very rare (accounting for about $0.02 \%$ ) and are usually present as sarcomas and lymphomas [4], whilst metastatic lesions are more common (accounting for approximately $21 \%$ cases; mainly including the pericardium by direct infiltration and less commonly, through metastasising via blood vessels or lymphatic routes). Clinical manifestations of secondary heart tumours are relatively late and are often accidentally recognised during imaging diagnostics, unfortunately, usually when vital structures of the heart have been affected [5].

The key issue underlying molecular target therapy is to block signal transfer which, in turn, adversely affects cellular division. This is usually achieved either by inactivating the ligand or blocking the receptor by specific antibody binding, blocking auto-phosphorylation or inhibiting the signalling of intracellular pathways. Cell cycle inhibitors and proteasome inhibitors may also be used. The mechanisms listed above, ie. signal pathways and/or kinase targets are also present within normal structures of the heart and/or the vascular system and therefore it is impossible to avoid targeted drugs interfering with healthy cardiomyocytes, that affect the function and the survival of myocardial cells [6].

\section{Cardiotoxic mechanisms of molecular target therapy}

The working experience of molecular target therapy acquired over the last few years has allowed two types of cardiovascular toxicity mechanisms to become identified:

1. Toxicity associated with specific mechanisms of action, ie. associated with the activation of an identical target point within the cardiomyocyte (e.g. kinase or receptors).

2. Toxicity associated with indirect or direct inhibition of other signalling pathways which, in effect, causes symptoms of cardiotoxicity (e.g. the affinity of sunitinib to block serotonin alpha and beta adrenergic receptors, the potassium channel protein receptor (hERG) and the Purkinje fibres, which is not observed in the case of sorafenib, which blocks only the hERG receptor protein and the Purkinje fibres) [7].

Table I shows the most common kinases which act as therapeutic targets for treating cancer and their potential effect on the cardiovascular system [8]. At present it is impossible to predict all potential forms of cardiotoxicity for molecular target therapy drugs because of the complicated nature of molecular targets. The vast majority of these drugs have multikinase effects and are characterised by different binding sites within the cardiomyocytes

\section{Cardiovascular disturbances}

\section{Hypertension}

One of the more common and critical clinical complications is arterial hypertension. It is usually associated whenever tyrosine kinase inhibitors (TKI) or anti-angiogenic drugs are given, such as bevacizumab. Among these drugs are also sunitinib, sorafenib and pazopanib. Their molecular action is based on blocking the internal domain of the vascular endothelial growth factor receptor (VEGFR). Bevacizumab has a slightly different mechanism of action, as it binds directly to the vascular endothelial growth factor (VEGF). TKls decrease the production of nitric oxide and thereby increase vascular resistance and blood flow deficits within capillaries. This may either cause arterial hypertension or exacerbate any pre-existing condition [9]. Murine studies have shown that mechanical factors are not important for inducing changes within large blood vessels, however the decrease in afterload brought on by TKIs directly affects the end-systolic resistance and eventually, the systolic function of the heart. Thus it can be ruled out that administering TKIs significantly decreases the'functional' density of capillaries.

Developing hypertension is also likely to be associated with renal changes. Animal studies have shown lesions within glomerular vessels, microangiopathic thrombosis, proliferation of the glomerular mesangium and excessive generation of fibrin. Normally, the activation of type 2 VEGFR activates phosphoinositide 3-kinase (PI3K)-AKT) and induces phosphorylation and activation of endothelial nitric oxides synthesis. This causes a secondary increase in guanylate cyclase activity and due to smooth muscle relaxation, blood vessels then dilate. During the production of nitric oxide, endothelin synthesis increases which causes vascular obturation. Unfortunately, the factor responsible for the endothelin synthesis increase remains unknown [10].

Most researchers agree that it is difficult to assess the number of patients with hypertension caused by TKI-treatment. One reason being that there are a number of definitions of hypertension applied according to the purposes of clinical trials. Also its measurement methods vary. The incidence of hypertension depends upon the type of drug. It is estimated that for all patients treated with TKIs, hypertension develops in $15 \%$ to $60 \%$ cases and is more serious as regards to systolic hypertension. In the instance of bevacizumab, arterial hypertension is observed in some $20-30 \%$ cases (of whom in 11-16\% cases it was observed for the first time). Severe hypertension is found in $8 \%$ cases, while hypertensive crisis in as many as $1 \%$ of subjects $[11,12]$. Chu et al. have shown arterial pressure to be $150 / 100 \mathrm{mmHg}$ or higher in $47 \%$ of patients during sunitinib therapy [13]. In a meta-analysis designed to assess the risk of hypertension in approximately 4600 patients treated for renal cancer with sorafenib, Wu reported grade 3 and 4 hypertension in 23\% 
Table I. The most common kinases and their potential effects on the cardiovascular system

\begin{tabular}{|c|c|}
\hline Kinase & Role of kinase in the cardiovascular system \\
\hline RAF1/ BRAF & Anti-apoptotic action, maintains the function of the left ventricle under increase load (stress) \\
\hline PI3K (p110a) & Physiologic heart growth, affects cardiomyocyte survival \\
\hline PI3K (p110y) & Regulation of contractility and pathological hypertrophy \\
\hline PDK1 & Affects cardiomyocyte survival and beta-adrenergic response \\
\hline Akt 1,2 or 3 & Regulation of cardiomyocyte survival, growth and metabolism \\
\hline mTOR & $\begin{array}{l}\text { Regulation of protein synthesis, inhibition causes energy preservation under duress; regulation of } \\
\text { protein synthesis; inhibition leads to energy saving under stress, regulation of Akt (mTORC2) activation } \\
\text { process }\end{array}$ \\
\hline AMPK & Supervision of energetic stress, mTOR1 inhibition while maintaining energy reserves \\
\hline GSK3 a/b & $\begin{array}{l}\text { mTORC1 inhibition (together with AMPK), GSKbeta deletion acts protectively in postinfarct remodelling; } \\
\text { GSKalfa deletion causes cardiac insufficiency under stress }\end{array}$ \\
\hline CDKs & $\begin{array}{l}\text { CDK2 inhibition limits heart damage in the course of ischemia-reperfusion acting through } \\
\text { retinoblastomy protein }\end{array}$ \\
\hline Aurora & Regulator of phase $\mathrm{M}$ of the cellular cycle \\
\hline PLKs & $\begin{array}{l}\text { Plk1 is involved in Cdc2 activation, chromosome segregation, centrosome maturation, forming of the } \\
\text { spindle apparatus and cytokines }\end{array}$ \\
\hline PDGFR & Participates in angiogenesis and in cardiac response to pressure stress (beta isoform) \\
\hline VEGFR & Participates in angiogenesis and in cardiac response to pressure stress (antihypertensive effect) \\
\hline EGFR (ErbB1) & $\begin{array}{l}\text { Preserving function of the; left ventricle under prolonged katecholamine stimulation; mediating the } \\
\text { signaling pathway stimulating cell survival }\end{array}$ \\
\hline ERBB2 ERBB4 & Influences the survival and homeostasis of cardiomyocytes and maintains function of left ventricle \\
\hline Kit & $\begin{array}{l}\text { Promotion of progenital cells in the heart and differentiation differentiation of immature } \\
\text { cardiomyocytes, direction towards post-ischaemic sites, promoting repair }\end{array}$ \\
\hline Abl/ARG & Maintenance of homeostasi, in rofents treated with imatinib left ventricular dysfunction is observed \\
\hline JAK2 & Together with STAT3 act protectively in many pathologic situations \\
\hline FAK & Activation decreases heart hypertrophy and fibrosis \\
\hline DMPK & $\begin{array}{l}\text { Type } 1 \text { myotonic dystrophy is brought on by additive repetitions of the } 3^{\prime} \mathrm{UTR} \text { regioon of the DMPK } \\
\text { kinase }\end{array}$ \\
\hline LTK & Activation causes hypertrophy and degeneration of cardiomyocytes \\
\hline ROCK & Activation of fibrosis and apoptosis under hypertensive stress \\
\hline LKB1 & Activation of AMPK which is proangiogenic within the heart \\
\hline LDB3, ZASP and/or Cypher & Induction of ZASP mutations associated with skeletal muscle myopathies \\
\hline ERK $1 / 2$ & Promotion of cardiomyocyte survival, ability to modulate physiologic (but not pathologic) hypertrophy \\
\hline PKC alfa & Destructive influence on the heart under hypertensive stress \\
\hline cGMP-dependent PK & $\begin{array}{l}\text { One of the four main kinases influencing cardiac failure; activated by PDE5 receptors; inhibits apoptosis, } \\
\text { hypertrophy and beta-adrenergic response }\end{array}$ \\
\hline PIM kinase & Influences the survival of cardiomyocytes (activated by Akt, regylated at the gene expression level) \\
\hline CAMKII & $\begin{array}{l}\text { Main kinase in cardiac failure; influences heart hypertrophy; promotes decompensation under } \\
\text { hypertensive stress }\end{array}$ \\
\hline GRK2 and/or GRK5 & Decrease in beta-adrenergic signalling through beta-arrestin recruitment \\
\hline ASK1 & Promotes pathologic hypertrophy and remodelling. Pro-apoptotic \\
\hline PTEN phosphatase & Inhibition of hypertrophy, decrease in cardiomyocyte survival under stress; PI3K antagonist \\
\hline
\end{tabular}

and $6 \%$ of subjects, respectively. The total risk of developing hypertension for sorafenib therapy was $6.11, \mathrm{p}<0.001$ [14]. Another meta-analysis on 5000 patients with gastrointestinal stromal tumours (GIST), demonstrated that the risk of developing hypertension was $22.72, p<0.001$ [15].
Many workers stress that during TKls administration, the rise in blood pressure may be rapid; especially in the case of sunitinib and sorafenib. This concerns patients who have not been previously treated for hypertension and may lead to a hypertensive crisis. 


\section{Prolonged QT interval}

The QT interval represents the time of ventricular repolarisation. Its length may be affected by many factors (e.g. age, autonomic stress, electrolyte levels, drugs or even the time of day). QT interval duration depends upon heart rhythm; during bradycardia it is long, in tachycardia it shortens. Measuring the QT interval is possible using a standard 12-channel ECG. In clinical practice, a correction of the QT interval is performed based upon heart rate, and a corrected value (QTc) is given.

Clinical symptoms of prolonged QT may cause syncope or a dangerous form of arrhythmia known as torsade de pointes which may cause ventricular fibrillation and eventually death. Many drugs administered as targeted therapy may prolong QT. Those include TKIs, histone deacetylase inhibitors, SRC/ABL inhibitors, FTpase, protein-Ckinase inhibitors, serine/threonine kinase protein-specific BRAF inhibitors and even inhibitors (blockers) of the type 3 serotonin receptor $[12,16,17]$.

\section{Prolonged QT interval - tyrosine kinase inhibitors}

In both preclinical and phase I trials of sunitinib in patients with solid tumours, prolonged repolarisation of the ventricular functional potential has been observed as represented by prolonged QT in a standard ECG. Changes in the QT interval of more than $60 \mathrm{~ms}$ (as compared to the initial values) were observed in $5 \%$ of the 84 subjects who had entered the study [18]. In a clinical trial involving 24 patients aged 24-87 years, the longest QT interval was seen on day 3, 24 hours after administering the drug (intended initial dose of $50 \mathrm{mg}$ ). This abnormality was not associated with any clinical problems. In none of the patients was the QT longer than $>500$ ms [19]. It is important that sunitinib has a binding affinity to $5 \mathrm{HT} 2$ a serotonin receptors, alpha and beta adrenergic receptors, hERG receptor protein and Purkinje fibres; blocking them all, which may affect repolarisation. Besides, electron microscopy investigations have shown both the presence of lesions and the activation of degenerative processes, particularly within the mitochondria as well as a significant decrease in the concentration of adenosine triphosphate (ATP) in the sunitinib and sorafenib groups [20].

Wandetanib, a tyrosine kinase inhibitor administered for treating medullary thyroid cancer in patients with the RETgene mutation, may significantly prolong ventricular repolarisation. A probable mechanism causing repolarisation disorders is interaction with ferrum channels within the heart. During a phase 1 study, QTc was prolonged in at least $10 \%$ of subjects; however this had no clinical implications and did not affect the course of drug administration [21]. In a phase II study of patients with breast cancer in whom wandetanib was administered $100 \mathrm{mg}$ per day together with docetaxel, QTC was prolonged in almost $5 \%$ of patients compared with the group receiving wandetanib $300 \mathrm{mg}$ per day with docetaxel, in whom prolonged QTc was observed in as many as $11 \%$ of subjects.

QTc prolongation was not observed in patients receiving docetaxel as monotherapy [22]. In a clinical study of wandetanib administered for non-small cell lung cancer, QTc was prolonged in $15 \%$ cases as compared to the non-wandetanib controls [23].

In a phase II trial of wandetanib on plasmocytoma patients there was also no QTc prolongation [24]. In another phase II wandetanib study on skin melanoma, it was found that during the first 6 months of treatment as many as 90 out of 132 patients had a QTc prolonged by $15.1 \mathrm{~ms}$ (in 2 patients, QTc exceeded $500 \mathrm{~ms}$, and in one case it reached $60 \mathrm{~ms}$ compared to the initial value) [25].

In view of such findings, it must be remembered that the cardiotoxicity seen for this group of drugs is probably triggered by a different mechanism and may be associated with a mitochondrial effect; it also arises from receptors being blocked to various extents within the cardiomyocytes. This might be the underlying issue responsible for the varied cardiotoxicity of TKIs.

\section{Prolonged QT interval - histone deacetylase inhibitors}

Deacetylases are regulatory proteins affecting the expression of genes dependent upon the presence of acetyl groups within histones. In many malignancies, acetylation abnormalities have been observed and this is the rationale behind attempts to apply these type of drugs for anticancer treatment [26].

Vorinostat and romidepsin have recieved FDA approval in the treatment of T-cell lymphoma of the skin, while the Committee for Medicinal Products for Human Use (CMHP) issued a negative opinion for romidepsin, stating that any clinical benefits have not been analysed (including longer overall survival). In the case of vorinostat, the submission for CMHP opinion had been withdrawn. In Europe therefore, histone deacetylase inhibitors are only administered in the course of clinical trials. Most of the data regarding their effects on the QT interval shows, that it is reversible and, usually of short duration. For the majority of patients, no symptomatic arrhythmias have been observed $[12,26]$.

\section{Prolonged QT interval — SRC/ABL inhibitors}

Common SRC/ABL inhibitors include nilotinib and dasatinib which are widely used in routine clinical practice.

Dasatinib is used for treating chronic myelogenous leukaemia $(\mathrm{CML})$ during the chronic phase in patients with the newly diagnosed disease who present with the Philadelphia chromosome ( $\mathrm{Ph}+$ ) as well as in cases of acute lymphoblastic leukaemia with $\mathrm{Ph}(+)$. In a phase II study on 
467 patients, the median QTc prolongation was between 3 and $6 \mathrm{msec}$ and in 64 patients the QTc was $60 \mathrm{~ms}$ longer as compared to initial values whilst in 3 patients the QTC was prolonged over $500 \mathrm{msec}$; this study demonstrating that exclusion criteria be applied also to patients with concomitant cardiovascular diseases. Thus, there seems to be a rationale behind the clinical observation that the hERG receptor within the heart is blocked 100 times less when compared to nilotinib [27].

Nilotinib may cause QT prolongation. In a clinical trial on patients resistant to imatinib, $4 \%$ of subjects presented with a QT pronged by $60 \mathrm{~ms}$ compared to baseline values. No prolongations over $500 \mathrm{~ms}$ were observed. Sudden death was observed in $0.6 \%$ cases and it was impossible to tell whether they were not associated with abnormal ventricular repolarisation $[28,29]$. It has been noted that other drugs which damage blood vessels, e.g. FTpase inhibitors and protein C kinase inhibitors (which are currently undergoing phase I trials), may potentially also prolong QTC [30].

\section{Cardiac ischemia}

Bevacizumab combined with standard chemotherapy may increase the number of emboli within the cardiovascular system and within the central nervous system. In a retrospective analysis, Scappaticci et al. showed a statistically significant risk of thromboembolism with combined therapy (standard + bevacizumab). The absolute risk of thromboembolic episodes reached 5.5 per 100 patient-years in the combined treatment group compared to 3.1 in the group receiving standard chemotherapy only. Risk factors included age over $65 \mathrm{yrs}$ and previous thromboembolic episodes [31]. Schimdinger reported thrombo-embolic incidents in $24 \%$ of patients treated with sunitinib [18]. In $17.6 \%$ of patients treated with sunitinib, a significant increase in the troponin level has been reported [13].

\section{Dysfunction of the left ventricle}

Different mechanisms underlie left ventricle dysfunction resulting from molecular target therapy. The best known is that associated with trastuzumab.

HER2 protein (ErB2) belongs to the family of epidermal growth factor receptors; EGFR. It is found on the malignant cells in some $20 \%$ of breast cancer patients. The protein consists of an external domain which binds the ligand, a transmembrane part and a cytoplasmatic part, which bears the characteristics of tyrosine kinase. The ErB2 receptor does not possess its own ligands, but it may undergo heterodimerisation with the remaining receptors, thus becoming activated.

Clinical trials have shown symptomatic and asymptomatic heart dysfunction in $4.7 \%$ of patients treated with trastuzumab only and in $27 \%$ of patients in whom trastuzumab is combined with anthracyclines. Risk factors of cardiotoxicity of trastuzumab include older age, previous treatment with anthracyclines and forms of heart injuries associated with concomitant diseases.

Under physiological conditions, activation of the Erb2 receptor within the heart is responsible for maintaining the correct structure and function of the myocardium. Binding with type 1 neuregulin (NRG1) activates the enzymatic pathway which determines the response to oxidative stress and initiates repairing mechanisms through the ERK and AKT routes. PI3K activation catalyses phosphorylation of the PK domain of AKT kinase, thus in turn initiating cellular mechanisms responsible for the growth and survival of myocytes. PI3K activation is possible through active RAS proteins. Additionally, NRG1 inhibition indirectly blocks the SRc and Fak routes, which exacerbates left ventricular dysfunction. Studies conducted on murine neonates in which the over-expression of antiapoptotic $\mathrm{BCl}-\mathrm{X}_{\mathrm{L}}$ cardiomyocyte protein has been genetically induced, has supported its protective role for developing dilation and hypertrophy of the myocardium on reaching adulthood [32-34].

It is important, that the type of cardiotoxicity induced by trastuzumab is classified as type II and reversible. The decrease in the ejection fraction is, in most cases, asymptomatic $[6,7,32]$. The HERA study has shown an asymptomatic decrease of the ejection fraction in $7 \%$ of patients, while symptomatic patients accounted for $1.7 \%$. In the NSABP B1 study, this ratio was $14.2 \%$ and $4.7 \%$, respectively (these differences arose mainly from qualification criteria and from methods used to assess cardiotoxicity) [35].

Ewer conducted a study in order to assess the clinical course of cardiotoxicity over a period of four years from the termination of anthracyclines treatment and under trastuzumab follow-up treatment [36].

The ejection fraction increased in $56 \%$ of patients after the withdrawal of trastuzumab and in $66 \%$ of cases treatment was recommenced. The median time for the ejection fraction to increase was 1.5 months. Only $12 \%$ of subjects developed left ventricle dysfunction.

A humanised monoclonal antibody which blocks the external subdomain of the ErbB2 receptor through inhibiting the dimerisation of the receptor, especially with the ErbB3 receptor, is pertuzumab which is yet another antibody which may be potentially useful for treating patients both with early and advanced breast cancer. Hitherto, studies have shown that for pertuzumab, cardiac failure is relatively rare and does not exceed a $1.2 \%$ rate in subjects. This difference is likely because of a different mechanism of action on the ErbB2 receptor. Within the cardiomyocyte, an important part of its protective mechanism is activating the ErbB2/ /ErbB4 dimer through NRG1 ligand binding to ErbB4. Pertuzumab inhibits the dimerisation process through inhibiting the ligand binding to the ErbB3 receptor [7]. 
When assessing the potential risk of developing cardiotoxicity (including heart failure associated with target therapy), one must always remember the different mechanisms of the two different molecular groups, that is the mAbs antibodies and the small molecules. Evidence for this has been provided by Spector et al, who has shown the possible protective function of lapatinib on the cardiomyocyte, which acts through the activation of a cardioprotective AMP-dependent kinase [37].

Published results of cardiovascular complication rates from randomised trials may actually show significantly different outcomes.

Populations qualified for treatment under pre-set and under 'normal' conditions vary significantly according to their cardiac status. A study by Tarantini shows that in 160 patients aged over 60 years, $32 \%$ had serious concomitant cardiovascular diseases. This is significantly more than in trial populations; the N-31 trial, the N9831 and the HEART of approximately $16 \%$ rates. Symptomatic cardiac failure was observed in $6 \%$ and in $2 \%$ before the age of 60 . Trastuzumab was stopped in $10 \%$ of patients aged over 60 years and in $4 \%$ of patients below 60 years, whilst treatment was recommenced in $44 \%$ of patients over 60 years and in $58 \%$ of patients aged below 60 .

Whenever $A B L$ inhibitors are used for treatment, it is assumed that left ventricular dysfunction for imatinib does not exceed $1 \%$. During electron microscopy investigations, myocyte necrosis was found; probably associated with apoptosis. It is likely that this mechanisms is similar to that observed in CML cells. Imatinib inhibits cellular processes responsible for deactivating oxidative stress, which become activated by the listed kinases.

Dilative cardiomyopathy was found in $4 \%$ patients treated with dasatinib and was caused by blocking the ABL/ /SRC route within the heart [12].

Cardiac incidents (including myocardial infarction, cardiac failure sudden cardiac death) are found in around $11 \%$ of GIST patients resistant to imatinib. In a retrospective study on a group of 224 patients, heart failure developed in $2.7 \%$ of patients and the median time to developing symptoms of cardiac failure was 22 days from the onset of sunitinib therapy, which supports the concept of the cumulative effect of sunitinib cardiotoxicity and its dependence on the duration of treatment [39]. It is estimated that cardiac failure, defined as a decrease in ejection fraction by over $15 \%$, occurs in about $9-18 \%$ of patients. In a clinical trial on 75 patients, Chu had shown a below $50 \%$ fall of the ejection fraction in $20 \%$ of patients; $8 \%$ of subjects having had symptoms of NYHA III or IV cardiac failure [13]. In $4.6 \%$ of patients studied by Telli, systolic dysfunction was seen; defined as a fall in ejection fraction below $20 \%$ of the initial value. The listed risk factors included previous cardiac disease (cardiac failure, angina) and low BMI values [40].
An interesting meta-analysis was prepared based upon the results published in the Medline database between January 1996 and February 2011. Subjects consisted of almost 7000 patients treated with sunitinib. In 45 cases, cardiac dysfunction was observed (in $1.5 \%$ of patients this was found to be significant), but no differences in the frequency of cardiac incidents were seen; neither depending on the type of disease nor on cardiac observation (renal cancer as compared to other malignancies; cardiac observation vs none) [41].

Pathologic features observed by electron microscopy showed cardiomyocyte hypertrophy with damaged mitochondria (oedema, altered structure, increased permeability of the mitochondrial membrane without necrosis nor intracellular tissue hypertrophy). Murine studies have only revealed cardiomyocyte necrosis in cases of arterial pressure stress induced by phenylephrine [20].

Numerous published observations from a significant number of clinical trials show that cardiac toxicity is usually reversible and disappears after treatment withdrawal in the majority of patients. However in some patients they may be severe, even resulting in death [17].

\section{Summary}

The use of modern diagnostic and therapeutic modalities has both significantly increased the number of complete recoveries and the survival of cancer patients. Nevertheless, the problem of treatment side-effects (incl. cardiovascular) remains a critical issue in oncology. This paper presents the most important issues of cardiotoxicity particularly regarding everyday practice of the oncologist. It also points out areas in which direct collaboration between the oncologist and the cardiologist may be necessary in order to provide optimal therapy for dealing with cardiac complications arising from oncological treatment.

\section{Conflict of interest: none declared}

\section{Beata Jagielska, PhD}

Department of Oncology Diagnostics, Cardioncology and Palliative Medicine

The Maria Skłodowska-Curie Memorial Cancer Center and Institute Roentgena 5

02-781 Warszawa, Poland

e-mail:bjagielska@coi.waw.pl

Received: 2 Feb 2016

Accepted: 27 Feb 2017

\section{References}

1. Eschenhagen T, Force T, Ewer MS et al. Cardiovascular side effects of cancer therapies: a position statement from the Heart Failure Association of the European Society of Cardiology. Eur J Heart Fail 2011; 13: 1-10.

2. Beukema JC, van Luijk P, Widder J et al. Is cardiac toxicity a relevant issue in the radiation treatment of esophageal cancer? Radiother Oncol 2015; 114: 85-90. 
3. Didkowska J, Wojciechowska U, Zatoński W. Nowotwory złośliwe w Polsce w 2011 roku. Warszawa: Centrum Onkologii — Instytut, 2013.

4. Sadowski J, Grudzień G. Nowotwory serca. In: Wielka interna - kardiologia zelementamiangiologii (part II). Pruszczyk P, Hryniewiecki T, Drożdż J (eds.). Warszawa: Medical Tribune, 2010: 606-619.

5. Plana JC, Galderisi M, Barac A et al. Expert consensus for multimodality imaging evaluation of adult patients during and after cancer therapy: a report from the American Society of Echocardiography and the European Association of Cardiovascular Imaging. Eur Heart J Cardiovasc Imaging 2014; 15: 1063-1093.

6. Siedlecki JA, Tudek B. Molekularne podstawy terapii ukierunkowanej na hamowanie przekaźnictwa wewnątrzkomórkowego. In: Leczenie ukierunkowane na cele molekularne w onkologii i hematoonkologii. Wojtukiewicz M, Sierko E (eds.).Gdańsk: Via Medica, 2013: 20-34.

7. Szmit S, Zaborowska M, Opolski G. Kardiotoksyczność terapii celowanej w onkologii i hematologii. In: Leczenie ukierunkowane na cele molekularne w onkologii i hematoonkologii. Wojtukiewicz M, Sierko E (eds.). Gdańsk: Via Medica, 2013: 536-551.

8. Force T, Kolaja KL. Cardiotoxicity of kinase inhibitors: the prediction and translation of preclinical models to clinical outcomes. Nat Rev Drug Discov 2011; 10: 111-126.

9. Chen M, Kerkelä R, Force T. Mechanisms of cardiac dysfunction associated with tyrosine kinase inhibitor cancer therapeutics. Circulation 2008; 118: 84-95.

10. Force T, Krause DS, Van Etten RA. Molecular mechanisms of cardiotoxicity of tyrosine kinase inhibiton. Nat Rev Cancer 2007; 7: 332-344.

11. Mourad JJ, des Guetz G, Debbabi H et al. Blood pressure rise following angiogenesis inhibition by bevacizumab. A crucial role for microcirculatin. Ann Oncol 2008: 19: 927-934.

12. Ederhy $\mathrm{S}$, Izzedine $\mathrm{H}$, Massard $\mathrm{C}$ et al. Cardiac side effects of molecular targeted therapies: towards a better dialogue between oncologists and cardiologists. Crit Rev Oncol Hematol 2011; 80: 369-379.

13. Chu TF, Rupnick MA, Kerkela $R$ et al. Cardiotoxicity associated with tyrosine kinase inhibitor sunitynib. Lancet 2007; 370: 2011-2019.

14. Wu S, Chen JJ, Kudelka A et al. Incidence and risk of hypertension with sorafenib in patients with cancer: a systematic review and meta-analysis. Lancet Oncol 2008; 9: 117-123.

15. Zhu X, Stergiopoulos K, Wu S. Risk of hypertension and renal dysfunction with an angiogenesis inhibitor sunitinib: systematic review and meta-analysis. Acta Oncol 2009; 48: 9-17.

16. Haverkamp W, Breithardt G, Camm AJ. The potential for QT prolongation and pro-arrhythmia by non-anti-arrhythmic drugs: clinical and regulatory implications. Report on a Policy Conference of the European Society of Cardiology. Cardiovasc Res 2000; 47: 219-233.

17. Mackey JR, Clemons M, Côté MA et al. Cardiac management during adjuvant trastuzumab therapy: recommendations of the he Canadian Trastuzumab Working Group. Curr Oncol 2008; 15: 24-35.

18. Schmidinger M, Zielinski CC, Vogl UM et al. Cardiac toxicity of sunitinib and sorafenib in patients with metastatic renal cell carcinoma. J Clin Oncol 2008; 26: 5204-5212.

19. BelloCL, Mulay M, Huang Xet al.Electrocardiographic characterization of the QTc interval in patients with advanced solid tumors: pharmacokinetic-pharmacodynamic evaluation of sunitinib. Clin Cancer Res 2009; 15: 7045-7052.

20. French K, Coatney RW, Renninger JP et al. Differences in effects on myocardium and mitochondria by angiogenic inhibitors suggest separate mechanisms of cardiotoxicity. Toxicol Pathol 2010; 38: 691-702.

21. Herbst RS, Heymach JV, O'Reilly MS et al. Vandetanib (ZD6474): an orally available receptor tyrosine kinase inhibitor that selectively targets pathways critical for tumor growth and angioenesis. Expert Opin Investig Drugs 2007; 16: 239-249.

22. Miller KD, Trigo JM, Wheeler C et al. A multicenter phase II trial of ZD6474, a vascular endothelial growth factor receptor- 2 and epidermal growth factor receptor tyrosine kinase inhibitor, in patients with previously treated metastatic breast cancer. Clin Cancer Res 2005; 11: 3369-3379.

23. Kiura K, Nakagawa K, Shinkai T et al. A randomized, double-blind, phase Ila dose-finding study of Vandetanib (ZD6474) in Japanese patients with non-small cell lung cancer. J Thorac Oncol 2008; 3: 386-393.
24. Kovacs MJ, Reece DE, Marcellus D et al. A phase II study of ZD6474 (Zactima), a selective inhibitor of VEGFR and EGFR tyrosine kinase in patients with relapsed multiple myeloma - NCIC CTG IND.145. Invest New Drugs 2006; 24: 529-535.

25. Charakterystyka produktu leczniczego: http://ec.europa.eu/health/ documents/community-register/2012/20120217116713/anx_116713_ pl.pdf.

26. Curigliano G, Cardinale D, Suter T et al.; ESMO Guidelines Working Group. Cardiovascular toxicity induced by chemotherapy, targeted agents and radiotherapy: ESMO Clinical Practice Guidelines. Ann Oncol 2012; 23 Suppl 7: 155-166.

27. US Food and Drug Administration (FDA):Dasatinib (BMS-35825) oncologic drug advisory comitee briefing document NDA 21-96. Washington DC:US Food and Drug Administration (FDA); 2006: 52.

28. le Coutre P, Ottmann OG, Giles F et al. Nilotinib (formerly AMN107) a highly selective $B C R-A B L$ tyrosine kinase inhibitor, is active in patients with imatinib-resistant or -intolerant accelerated-phase chronic myelogenous leukemia. Blood 2008; 111: 1834-1839.

29. Kantarjian HM, Giles F, Gatermann $\mathrm{N}$ et al. Nilotinib (formerly AMN 107), a highly selective $B C R-A B L$ tyrosine kinase inhibitor, is effective in patients with Philadelphia chromosome-positive chronic myelogenous leukemia in chronic phase following imatinib resistence and intolerance. Blood 2007; 110: 3540-3546.

30. Britten CD, Rowinsky EK, Soignet S et al. A phase I and pharmacological study of the farnesyl protein transferase inhibitor L-778,123 in patients with solid malignancies. Clinical Cancer Res 2001; 7: 3894-3903.

31. Scappaticci FA, Skillings JR, Holden SN et al. Arterial thromboembolic events in patients with metastatic carcinoma treated with chemotherapy and bevacizumab. J Nat/ Cancer Inst 2007; 99: 1232-1239.

32. Zeglinski M, Ludke A, Jassal DS et al. Trastuzumab-induced cardiac dysfunction: A "dual-hit". Exp Clini Cardiol 2011; 16: 70-74.

33. Piotrowski G, Gawor R, Gawor Z et al. Role of echocardiography in monitoring of cardiac toxicity of cancer pharmacotherapy. Expert consensus statement of the Polish Clinical Forum for Cardiovascular Imaging. Kardiol Pol 2014; 72: 558-575.

34. Opolski G, Krzakowski M, Szmit S et al. Recommendations of National Team of Cardiologic and Oncologic Supervision on cardiologic safety of patients with breast cancer. The prevention and treatment of cardiovascular complications in breast cancer. The Task Force of National Consultants in Cardiology and Clinical Oncology for the elaboration of recommendations of cardiologic proceeding with patients with breast cancer. Kardiol Pol 2011; 69: 520-530.

35. Romond $\mathrm{EH}$, Jeong JH, Rastogi $\mathrm{P}$ et al. Seven-year follow-up assessment of cardiac function in NSABP B-31, a randomized trial comparing doxorubicin and cyclophosphamide followed by paclitaxel (ACP) with ACP plus trastuzumab as adjuvant therapy for patients with node-positive, human epidermal growth factor receptor 2-positive breast cancer. J Clin Oncol 2012; 30: 3792-3799.

36. Ewer MS, Vooletich MT, Durand JB et al. Reversibility of trastuzumab-related cardiotoxicity: New insights based on clinical course and response to medical treatment. J Clin Oncol 2005; 23: 7820-7827.

37. Spector NL, Yarden Y, Smith B et al. Activation of AMP-activated protein kinase by human EGF receptor 2/EGF receptor tyrosine kinase inhibitor protects cardiac cells. Proc Natl Acad Sci USA 2007; 104: 10607-10612.

38. Tarantini L, Gori S, Faggiano P et al. Adjuvant trastuzumab cardiotoxicity in patients over 60 years of age with early breast cancer: a multicenter cohort analysis. Ann Oncol 2012; 23: 3058-3063.

39. Khakoo AY, Kassiotis CM, Tannir $\mathrm{N}$ et al. Heart failure associated with sunitinib malate: a multitargeted receptor tyrosine kinase inhibitor. Cancer 2008; 112: 2500-2508.

40. Telli ML, Witteles RM, Fisher GA et al. Cardiotoxicity associated with the cancer therapeutic agent sunitinib malate. Ann Oncol 2008; 19: 1613-1618.

41. Richards $C J$, Je Y, Schutz FA et al. Incidence and risk of congestive heart failure in patients with renal and nonrenal cell carcinoma treated with sunitinib. J Clin Oncol 2011; 29: 3450-3456. 